\title{
Efeitos da informação comportamental na dinâmica intrínseca do controle postural
}

\author{
Alaércio Perotti Junior ${ }^{1}$ \\ José Angelo Barela ${ }^{2}$ \\ Paula Favaro Polastri ${ }^{3}$ \\ Go Tani ${ }^{4}$ \\ ${ }^{1}$ Centro Universitário Herminio Ometto de Araras, SP, Brasil \\ ${ }^{2}$ Departamento de Educação Física, IB/UNESP - Universidade Estadual Paulista, Rio Claro, SP, Brasil \\ ${ }^{3}$ Departamento de Educação Física, Faculdade de Ciências de Bauru, Universidade Estadual Paulista, \\ Bauru, SP, Brasil \\ ${ }^{4}$ Departamento de Pedagogia do Movimento do Corpo Humano, Escola de Educação \\ Física e Esporte, Universidade de São Paulo, SP, Brasil
}

\begin{abstract}
Resumo: Esse estudo teve como objetivo examinar possíveis alterações na dinâmica intrínseca de crianças e adultos decorrentes de informações externas na realização de uma tarefa de manutenção da postura ereta. Participaram do estudo dez crianças de 8 anos de idade e dez adultos jovens de ambos os gêneros. Eles permaneceram na posição ereta dentro de uma sala móvel que foi movimentada continuamente para frente e para trás. Os participantes recebiam informação sobre o movimento da sala e eram solicitados a não oscilar ou a oscilar junto com o movimento da mesma. Os resultados mostraram que a manipulação da informação visual induziu oscilação corporal correspondente (dinâmica intrínseca) em crianças e adultos. Informação sobre o movimento da sala e solicitação de uma ação (informação comportamental) alteraram o relacionamento entre informação visual e oscilação corporal. Crianças apresentaram mais dificuldades em alterar a dinâmica intrínseca do que adultos, indicando que elas são mais dependentes da dinâmica intrínseca do que adultos. Esses resultados trazem implicações importantes para a situação de ensino-aprendizagem, pois indica que aprendizagem envolvendo crianças deve ser estruturada propiciando condições mais favoráveis para alterações na dinâmica intrínseca para que os objetivos da mesma sejam alcançados.
\end{abstract}

Palavras-chave: Dinâmica intrínseca. Informação comportamental. Informação. Controle motor.

\section{Behavioral information effects on postural control intrinsic dynamics}

\begin{abstract}
The purpose of this study was to examine possible changes in the intrinsic dynamics of children and adults resulting from external information in upright stance task. Ten eight-year-old children and ten young adults, both genders, maintained upright position inside a moving room that was continuously moved frontward and backward. Participants were informed about the room movement and they were requested to sway or not sway with the moving room. The results showed that visual manipulation induces correspondent body sway (intrinsic dynamics) in children and adults. Information about the room movement and the request of an action (behavioral information) changed the relation between visual information and body sway. Although it can be changed, children showed more difficulty in changing the intrinsic dynamics than adults, showing that they are more dependent on intrinsic dynamics than adults. These findings bring important implications to learning, suggesting that children's learning opportunities must be structured properly in order to provide condition of intrinsic dynamics changes towards the goals of the task at hand.
\end{abstract}

Keywords: Intrinsic dynamics. Behavioral information. Information. Motor control. Posture.

\section{Introdução}

A execução de uma ação motora requer a produção de atividade motora específica e o uso de informação sensorial, que em muitos casos ocorrem sem a necessidade de um dispêndio de atenção e conhecimento discriminatório por parte do executante. Tal mecanismo é muito importante, pois possibilita que recursos de atenção sejam direcionados para outras tarefas que àquelas de controlar um movimento específico. Schöner e Kelso (1988a) sugeriram que a ocorrência desse tipo de comportamento refletiria a dinâmica de funcionamento do sistema sensório-motor, denominada de dinâmica intrínseca. Neste caso, dinâmica intrínseca referese a padrões comportamentais que o sistema tem preferência em exibir e define a ocorrência de comportamentos estáveis que um dado sistema espontaneamente adota na ausência de exigências ambientais específicas (Zanone e Kelso, 1992). 
Por outro lado, um sistema não pode apenas realizar comportamentos preferidos, pois seria incapaz de se adaptar às exigências e demandas impostas pelas condições ambientais, que em muitos casos requerem comportamentos diferentes daqueles preferidos e definidos pela dinâmica intrínseca. Assim, Schöner e Kelso (1988b) sugeriram que comportamentos preferidos podem ser alterados a partir de imposições ambientais, denominadas de informação comportamental. Especificamente, informação comportamental é qualquer restrição imposta ao funcionamento de um sistema de forma que a atuação do mesmo ocorra em regimes diferentes do preferido (Zanone e Kelso, 1992).

Um exemplo de dinâmica intrínseca e informação comportamental pode ser observado no controle postural. Na posição em pé, por exemplo, a frequência preferida de oscilação corporal é ao redor de $0,2 \mathrm{~Hz}$ (Soames e Atha, 1982), e essa oscilação reflete a dinâmica intrínseca de funcionamento do sistema de controle postural. Porém, essa não é a única frequência de oscilação e tanto adultos quanto crianças exibem oscilação corporal em outras frequências, quando informação sensorial é manipulada (Barela et al., 2001; Barela et al., 2003). Neste caso, a manipulação da informação sensorial constitui uma fonte de informação comportamental, decorrente de manipulação de estímulos disponíveis no ambiente, que altera a dinâmica intrínseca de funcionamento do sistema de controle postural.

Lee e colaboradores (Lishman e Lee, 1973; Lee e Aronson, 1974; Lee e Lishman, 1975) implementaram essa estratégia para verificar os efeitos da manipulação de estímulos visuais no controle postural. Neste caso, participantes mantinham a postura ereta no interior de uma sala em que as paredes laterais e do teto podiam ser movimentadas para frente e para trás, independentemente do piso onde eles eram posicionados. A movimentação das paredes da sala provocou alteração no fluxo óptico e oscilações corporais foram desencadeadas na mesma direção do movimento da sala. Esse acoplamento entre informação visual e oscilação corporal pode refletir a dinâmica intrínseca do sistema de controle postural, pois 0 funcionamento do sistema é alterado e produz oscilação corporal acompanhando o movimento da sala. Por outro lado, informação fornecida sobre o movimento da sala ou alguma solicitação para alterar este relacionamento é considerada informação comportamental, pois constitui uma exigência para alterar 0 relacionamento entre a informação visual e a oscilação corporal durante a manutenção da posição ereta na sala móvel.

O paradigma da sala móvel tem sido extensamente utilizado para verificar diversos aspectos do uso da informação visual no controle postural de bebês e crianças (e.g., Butterworth e Hicks, 1977; e.g., Bertenthal e Bai, 1989; Delorme, Frigon et al., 1995; Higgins, Campos et al., 1996; Bertenthal et al., 1997). Estes estudos têm demonstrado que mesmo bebês são susceptíveis à manipulação da informação visual, tanto com movimentos discretos quanto com movimentos contínuos da sala móvel, e que mudanças de desenvolvimento associadas às experiências motoras propiciam a ocorrência de respostas mais coerentes e estáveis quando ocorre manipulação da informação visual.

Além de indicar a estreita relação entre informação sensorial e oscilação corporal, estudos têm utilizado o mesmo paradigma para entender os mecanismos adjacentes deste relacionamento em bebês e crianças (Schmuckler, 1997; Barela et al., 2000) e para obter possíveis explicações das mudanças que ocorrem no controle postural (Barela et al., 2003; Godoi e Barela, 2008; Rinaldi et al., 2009). Schmuckler (1997) observou que crianças mesmo de três anos de idade conseguiam acoplar suas oscilações corporais às diferentes frequências de oscilação da sala móvel, como consequência do fluxo óptico. Resultados semelhantes foram observados por Barela et al. (2003), utilizando uma versão da sala móvel para a manipulação da informação somatosensorial. Entretanto, estes autores observaram que mesmo crianças de oito anos de idade ainda apresentavam um acoplamento entre informação sensorial e ação motora mais instável e variado que o observado em adultos. Com base nesses resultados, foi sugerido que diferenças no funcionamento do sistema de controle postural seriam decorrentes de dificuldades que crianças teriam em estimar a dinâmica corporal (posição e velocidade de deslocamento do corpo) e em enviar comandos precisos para a ativação da musculatura responsável pela manutenção da posição corporal desejada (Barela et al., 2003). Mais recentemente, tem sido sugerido que a 
dificuldade em obter um relacionamento entre informação sensorial e oscilação corporal com a mesma estabilidade observada em adultos seria decorrente do fato de que crianças não conseguem obter estímulos sensoriais com a mesma propriedade informativa que adultos conseguem quando os estímulos sensoriais são alterados (Godoi e Barela, 2008; Rinaldi et al., 2009).

Com base nesses resultados, pode-se inferir que crianças podem ter a dinâmica intrínseca de funcionamento do sistema de controle postural alterada por alguma manipulação de informação sensorial (Schmuckler, 1997; por ex., Godoi e Barela, 2008). Mais ainda, parece que mudanças no desenvolvimento são decorrentes de como elas utilizam informação sensorial para produzir ativação muscular apropriada e assim manter 0 corpo em uma dada orientação postural (Peterson et al., 2006; Godoi e Barela, 2008; Rinaldi et al., 2009). Finalmente, o funcionamento do sistema de controle postural de crianças é influenciado por informação comportamental disponível, entretanto, não de forma estável como observada em adultos, pela dificuldade em extrair as informações mais adequadas a partir dos estímulos disponíveis (Barela et al., 2003), de forma a manter um relacionamento coerente entre estímulos sensoriais disponíveis e oscilação corporal durante a manutenção da postura ereta.

Com base nestas proposições, há
necessidade de ampliar o escopo das
investigações incluindo não apenas as
manipulações sensoriais, mas também
informação sobre 0 contexto ambiental. Informação sobre o que estaria acontecendo no contexto ambiental afetaria o relacionamento entre informação sensorial e ação motora? Em estudo recente, adultos expostos à manipulação visual na sala móvel conseguiram utilizar informação sobre o movimento da mesma, entretanto, em condições em que as características do estímulo eram alteradas, a obtenção dessa informação ocorria de forma automática (Barela, Barela et al., 2009). Ainda, há diferença entre apresentar informação ou solicitar uma ação específica na alteração da dinâmica intrínseca de relacionamento entre informação visual e oscilação corporal?

A utilização de informação apresentada sobre o contexto ou a solicitação para realizar uma ação simula situações vivenciadas durante o processo de aprendizagem ou de execução de uma tarefa motora. Aprendizagem ocorre a partir de contexto específico, cunhado a partir das experiências anteriores (Zanone e Kelso, 1992; Barela e Barela, 2001). Assim, o aprendiz inicia a realização de uma determinada tarefa tendo como ponto de partida certa dinâmica intrínseca de funcionamento do sistema perceptivo motor e o professor/instrutor tem o papel de ser uma fonte de informação comportamental que, com suas instruções, procura alterar a execução daquela tarefa (Schöner et al., 1992; Zanone e Kelso, 1992; Barela e Barela, 2001; Newell, 2003). Nesse quadro, cabe perguntar se a dinâmica intrínseca de funcionamento do sistema pode ser alterada pela instrução verbal fornecida às crianças por um agente externo. Nesse sentido, espera-se que a dinâmica intrínseca seja alterada pela instrução ou solicitação, por exemplo, fornecida por outra pessoa.

Schöner et al. (1998) sugeriram que o sistema de controle postural pode diminuir o acoplamento entre informação sensorial e ação motora e alterar a dinâmica intrínseca com base em informação sobre o contexto ambiental. Essa sugestão foi testada em estudo com adultos, quando informação sobre o movimento da sala móvel foi fornecida aos participantes (Freitas Júnior e Barela, 2004) e quando eles foram solicitados a resistir à influência da manipulação da sala móvel (Stoffregen et al., 2006; Barela, Barela et al., 2009). Mais ainda, é pertinente indagar se para crianças, no caso em que elas obtêm informação comportamental para alterar a dinâmica intrínseca do sistema diferentes tipos de instrução sobre a situação ou uma determinada ação - têm efeitos diferentes na dinâmica intrínseca de funcionamento do sistema. Recentemente, testamos como crianças usam informação comportamental para alterar a dinâmica intrínseca de funcionamento em uma tarefa discreta de controle postural (Perotti Jr. et al., 2007). Os resultados indicaram que crianças conseguem alterar a dinâmica intrínseca do relacionamento entre informação visual e oscilação corporal, entretanto, não de forma tão precisa quanto os adultos. Embora importantes, estes resultados foram obtidos a partir de uma manipulação discreta da informação visual (um único movimento da sala) e há necessidade de verificar se tais resultados também são observados em situações em que 0 relacionamento entre informação sensorial e ação 
motora ocorre de forma repetitiva ao longo de um período de tempo (movimento contínuo e oscilatório da sala móvel).

Assim, esse estudo teve como objetivo examinar possíveis alterações na dinâmica intrínseca de crianças e adultos decorrentes de informações externas na realização de uma tarefa de manutenção da postura ereta. Especificamente, o objetivo foi examinar os efeitos de apresentar informação sobre o contexto ambiental (movimento da sala) e de solicitar uma ação motora específica (não oscilar e oscilar junto com a sala) no relacionamento entre informação visual e oscilação corporal em crianças e adultos durante a manutenção da postura ereta.

\section{Participantes}

\section{Método}

Participaram desse estudo dez crianças de 8 anos de idade $(M=95,5$ meses, $D P=4,8)$ e dez adultos jovens $(M=278,0$ meses, $D P=46,3)$ de ambos os gêneros. Eles foram recrutados na comunidade de Rio Claro e Limeira, mais especificamente, em escolas públicas, centros comunitários, familiares e alunos de graduação do Instituto de Biociências, UNESP, Campus de Rio Claro. Todos os participantes possuíam visão normal e não apresentavam nenhum comprometimento motor que pudesse afetar a manutenção da postura ereta. Os voluntários adultos assinaram um termo de concordância para participar do estudo, aprovado pelo Comitê de Ética da Escola de Educação Física e Esporte da Universidade de São Paulo - SP. No caso das crianças, o termo foi assinado por um dos pais ou responsável.

\section{Procedimentos}

O experimento foi realizado no Laboratório para Estudos do Movimento (LEM), do Departamento de Educação Física, IB, UNESP Rio Claro e no Laboratório de Comportamento Motor (LACOM) da Escola de Educação Física e Esporte USP - São Paulo. Os participantes fizeram uma visita ao LEM, quando os objetivos e procedimentos foram explicados e, caso concordassem em participar do estudo, o termo de consentimento foi assinado.

A tarefa experimental foi permanecer dentro de uma sala móvel na postura ereta estática, descalços, mantendo os pés paralelos distantes aproximadamente $3 \mathrm{~cm}$ entre eles, com os braços ao lado do corpo, olhando para um alvo fixado na parede do fundo da sala e na altura de seus olhos. A sala é constituída de três paredes e um teto com 2,1 x 2,1 x 2,1 metros (altura, largura $e$ comprimento). Estas paredes são de cor branca com faixas pretas verticais em toda a sua extensão, com o objetivo de aumentar o contraste visual. A estrutura que sustenta a parede e o teto tem, na parte inferior, rodas posicionadas sobre trilhos. Esta constituição possibilita o movimento das paredes da sala independente do piso no qual o participante está posicionado, que permanece fixo.

Os movimentos da sala foram produzidos e controlados por um sistema de servo-motor, comandado por programas computacionais específicos (Motion Architect for Windows Compumotor - APEX6151), que envia sinais quanto à amplitude, velocidade e aceleração do movimento a ser executado. Os movimentos desta sala foram realizados para frente e para trás, de forma contínua, o que correspondeu aos movimentos da parede frontal de se aproximar e se afastar do participante, respectivamente. Sendo assim, estes movimentos foram realizados na direção ântero-posterior em relação ao posicionamento do participante.

Foram realizadas 18 tentativas com duração de 60 segundos cada. O Quadro 1 apresenta as condições, a informação fornecida e a ação solicitada aos participantes. Como pode ser observado, as 18 tentativas foram divididas em três blocos de seis tentativas cada. Em cada bloco, foram realizadas três tentativas com movimento da sala e três tentativas sem movimento da sala. No primeiro bloco, os participantes não receberam qualquer informação sobre o movimento da sala, sendo apenas solicitados que permanecessem o mais estático possível. No segundo bloco, os participantes foram informados que a sala seria movimentada para frente e para trás sendo essa informação falsa para aquelas tentativas em que a sala não foi movimentada e verdadeira naquelas em que a sala foi movimentada. As ações solicitadas eram para que 0 participante permanecesse estático (tentativas sem movimento da sala) ou oscilasse junto com a sala (tentativas com movimento da sala). No terceiro bloco, os participantes receberam a mesma informação fornecida no segundo bloco, entretanto, a ação solicitada foi oposta ao segundo bloco. Assim, nas tentativas em que a sala não era movimentada, a ação solicitada aos participantes era para que eles 
oscilassem junto com a sala e nas tentativas em que a sala era movimentada, que não oscilassem com a sala. A ordem das tentativas, dentro de cada bloco, foi determinada previamente mediante sorteio realizado antes do início dos procedimentos experimentais para cada participante.

Quadro 1. Representação esquemática da movimentação da sala de forma contínua, informação fornecida sobre o movimento da sala e ação solicitada ao sujeito.

\begin{tabular}{|c|c|c|}
\hline Movimento da Sala & $\begin{array}{c}\text { Informação sobre movimento da } \\
\text { sala }\end{array}$ & Ação solicitada \\
\hline Sem Movimento & Nenhuma & Não Oscile - Permaneça Estático \\
\hline Sem Movimento & Nenhuma & Não Oscile - Permaneça Estático \\
\hline Sem Movimento & Nenhuma & Não Oscile - Permaneça Estático \\
\hline Com Movimento & Nenhuma & Não Oscile - Permaneça Estático \\
\hline Com Movimento & Nenhuma & Não Oscile - Permaneça Estático \\
\hline Com Movimento & Nenhuma & Não Oscile - Permaneça Estático \\
\hline \multicolumn{2}{|c|}{ Intervalo } \\
\hline Sem Movimento & Falsa & Não Oscile - Permaneça Estático \\
\hline Sem Movimento & Falsa & Não Oscile - Permaneça Estático \\
\hline Sem Movimento & Falsa & Não Oscile - Permaneça Estático \\
\hline Com Movimento & Correta & Oscile junto com a sala \\
\hline Com Movimento & Correta & Oscile junto com a sala \\
\hline Com Movimento & Correta & Oscile junto com a sala \\
\hline \multicolumn{2}{|c|}{ Intervalo } & Oscile junto com a sala \\
\hline Sem Movimento & Falsa & Oscile junto com a sala \\
\hline Sem Movimento & Falsa & Oscile junto com a sala \\
\hline Sem Movimento & Falsa & Não Oscile - Permaneça Estático \\
\hline Com Movimento & Correta & Não Oscile - Permaneça Estático \\
\hline Com Movimento & Correta & Não Oscile - Permaneça Estático \\
\hline Com Movimento & Correta
\end{tabular}

Nas tentativas em que ocorreu o movimento da sala, a mesma foi movimentada de forma contínua, para frente e para trás, na frequência de $0,2 \mathrm{~Hz}$, com amplitude de $1,8 \mathrm{~cm}$ e com uma velocidade de pico constante de $0,9 \mathrm{~cm} / \mathrm{s}$. A escolha da frequência de $0,2 \mathrm{~Hz}$ para a movimentação da sala se deu por estar próxima à frequência natural de oscilação de adultos (Soames e Atha, 1982) e de crianças (Godoi e Barela, 2008) durante a manutenção da posição em pé. A amplitude e velocidade da sala ficaram acima de estudos prévios (Freitas Júnior e Barela, 2004; Barela, Barela et al., 2009), porém ainda sem que os participantes conseguissem detectar conscientemente o movimento da sala.

Para obter informação sobre os movimentos da sala móvel e da oscilação corporal dos participantes foi utilizado um sistema de análise tridimensional de movimento optoeletrônico (OPTOTRAK 3020 - Northern Digital Inc.). Esse sistema é constituído de uma unidade de controle, uma unidade de sensores e emissores infravermelhos. Um emissor infravermelho foi afixado na parede frontal da sala e outro nas costas do participante ( $8^{\underline{a}}$ vértebra torácica, entre as escápulas). Esses emissores, em conjunto com a unidade de controle e os sensores, forneceram informação sobre a posição da sala e do participante nas direções ântero-posterior, médio-lateral e vertical ao longo da tentativa. A frequência de aquisição desses sinais foi de 100 $\mathrm{Hz}$.

O relacionamento entre o movimento da sala e a oscilação corporal frente ao fornecimento de informação verbal foi analisado pela utilização das medidas coerência e ganho (Jeka et al., 1997; Barela et al., 2003). A coerência indica o quão dependente dois sinais estão, neste caso, a posição da sala móvel e a posição corporal. Valores de coerência próximos de 1 e de zero indicam forte e fraca dependência, respectivamente, entre o movimento da sala e a oscilação corporal. O ganho indica o quão a oscilação corporal foi influenciado pela oscilação da sala, sendo calculado como a razão da amplitude espectral entre os dois sinais (Jeka et al., 1997). Valores próximos de 1 indicam que a oscilação corporal apresentou a mesma amplitude que a oscilação da sala. Valores menores e 
maiores que 1 indicam que a oscilação corporal apresentou amplitude menor e maior que a sala, respectivamente. Valores de coerência e ganho foram calculados na frequência do estímulo, na frequência de $0,2 \mathrm{~Hz}$. Para cálculo destas variáveis nas condições em que a sala permaneceu estacionária, foram utilizados dados sobre a posição da sala móvel de uma tentativa em que a sala foi movimentada.

Finalmente, foi calculada a amplitude média de oscilação corporal em todas as tentativas, indicando a magnitude de oscilação corporal geral, obtida a partir dos dados do emissor posicionado nas costas do participante na direção ântero-posterior. A amplitude média de oscilação foi calculada subtraindo um polinômio de primeira ordem e a média de todos os sinais da tentativa. Posteriormente, o desvio padrão destes sinais foi calculado, indicando assim a variabilidade da oscilação corporal em uma tentativa. Desta forma, valores de amplitude média de oscilação indicaram menor e maior magnitude de oscilação corporal, respectivamente.

\section{Análise estatística}

Quando nenhuma informação verbal foi fornecida, foram utilizadas uma análise de variância (ANOVA) e uma análise de multivariância (MANOVA), tendo como fatores grupo (crianças e adultos) e condição de movimento da sala (sem e com movimento), sendo este último fator tratado como medidas repetidas. A variável dependente para a ANOVA foi a amplitude média de oscilação e as variáveis dependentes para a MANOVA foram os valores de coerência e ganho.

Ainda, foram realizadas uma ANOVA e uma MANOVA, tendo como fatores grupo (crianças e adultos), condição de movimento da sala (sem movimento e com movimento) e ação solicitada (não oscilar e oscilar junto com a sala). A ANOVA teve como variável dependente a amplitude média de oscilação corporal e a MANOVA teve como variáveis dependentes a coerência e o ganho.

Quando houve necessidade, testes de análises univariadas e testes "post hoc" utilizando ajustes de Bonferroni foram realizados. Todas as análises estatísticas foram realizadas utilizando o programa SPSS (SPSS para Windows - versão 6.1) e o valor de alfa foi de 0,05 .

\section{Resultados}

Comportamento sem fornecimento de informação e solicitação verbal (dinâmica intrínseca)

A Figura 1 apresenta a amplitude média de oscilação para ambos os grupos sem e com o movimento da sala. A ANOVA revelou diferença para grupo, $F(1,18)=4,954, p<0,05$, e movimento, $F(1,18)=52,751, \quad p<0,001$, porém nenhuma interação entre grupo e movimento, $F(1,18)=0,266, p>0,05$. As crianças apresentaram oscilação corporal maior que os adultos e ambos os grupos oscilaram mais quando a sala foi movimentada do que quando não ocorreu movimento da mesma.

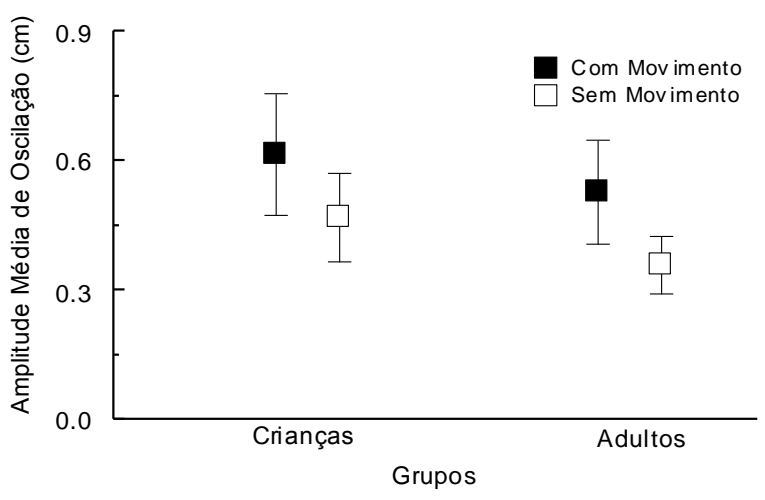

Figura 1. Média e desvio-padrão da amplitude média de oscilação para os grupos de crianças e adultos nas condições com e sem movimento da sala e sem qualquer informação.

O relacionamento espacial entre a posição da sala e a oscilação corporal foi examinado a partir das variáveis coerência e ganho que são apresentadas para ambos os grupos e nas condições com e sem movimento na Figura 2. A MANOVA revelou diferença apenas para 0 fator movimento, Wilks`'Lambda $=0,081, F(2,17)=96,73$, $\mathrm{p}<0,001$. Análises univariadas revelaram diferenças para coerência, $F(1,18)=200,08$, $p<0,001$, e ganho, $F(1,18)=97,44, \quad p<0,001$. Quando a sala foi movimentada, os valores de coerência e de ganho foram maiores para ambos os grupos do que quando a sala permaneceu sem movimento. 

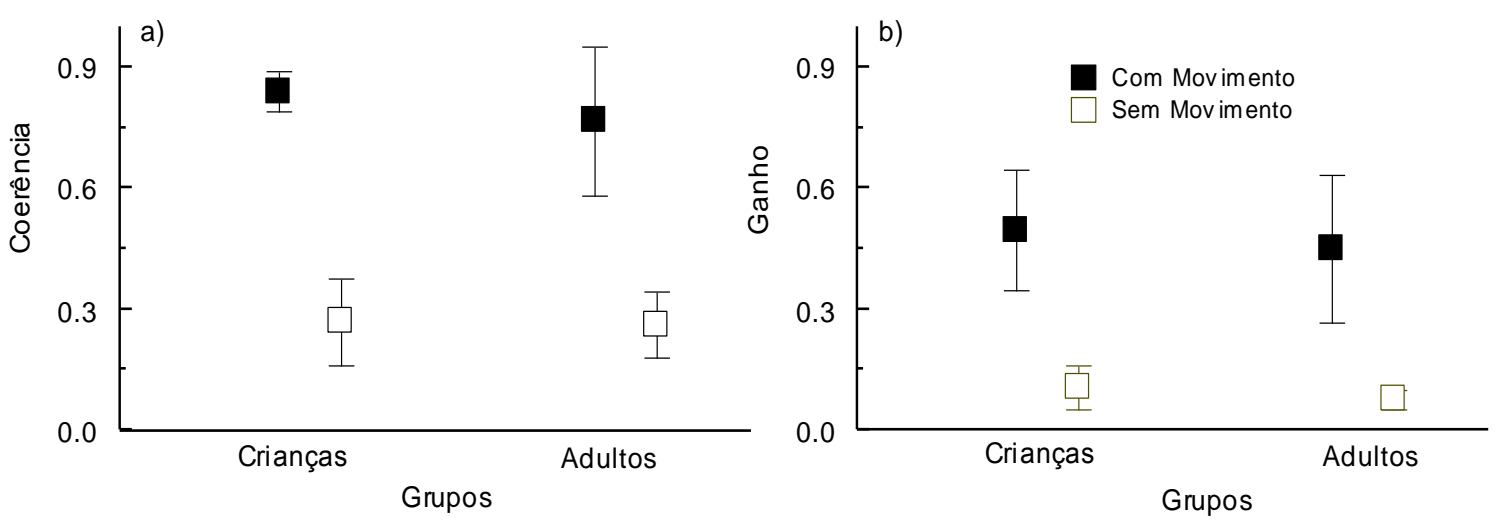

Figura 2. Média e desvio-padrão de coerência (a) e de ganho (b) para os grupos de crianças e adultos nas condições com e sem movimento da sala e sem qualquer informação.

Comportamento com fornecimento de informação e solicitação verbal (informação comportamental)

O comportamento dos participantes frente ao fornecimento de informações corretas e falsas e da ação de não oscilar e oscilar junto com a sala foi verificado através da variável amplitude média de oscilação. A Figura 3 apresenta os valores médios e respectivos desvios padrão da amplitude média de oscilação nas duas condições, com fornecimento de informação verbal correta e falsa, para os dois grupos etários, na ação de não oscilar e oscilar junto com a sala. Ambos os grupos apresentaram uma amplitude de oscilação semelhante quando a ação solicitada era não oscilar, em ambas as condições, com informação verbal correta e falsa. A ANOVA revelou diferença para grupo, $F(1,18)=11,416$, $p<0,005$, informação, $F(1,18)=31,10, \quad p<0,001$, ação, $F(1,18)=34,51, p<0,001$, interação entre informação e ação, $F(1,18)=21,68, p<0,001$, e grupo, informação e ação, $F(1,18)=5,85, p<0,05$. Analisando a interação tripla, percebe-se que os adultos conseguiram realizar as ações solicitadas e discriminar as condições de informação correta e falsa. Neste caso, os adultos não oscilaram ou oscilaram juntos com o movimento da sala na condição de informação correta, porém na condição de informação falsa praticamente não oscilaram, apesar de serem solicitados a fazer, quando a sala não foi movimentada, mesmo tendo sido informados que a mesma seria movimentada. Diferentemente, as crianças realizaram as ações solicitadas (não oscilar e oscilar junto) similarmente com o fornecimento de informação correta e falsa e oscilando mesmo quando a sala não foi oscilada.
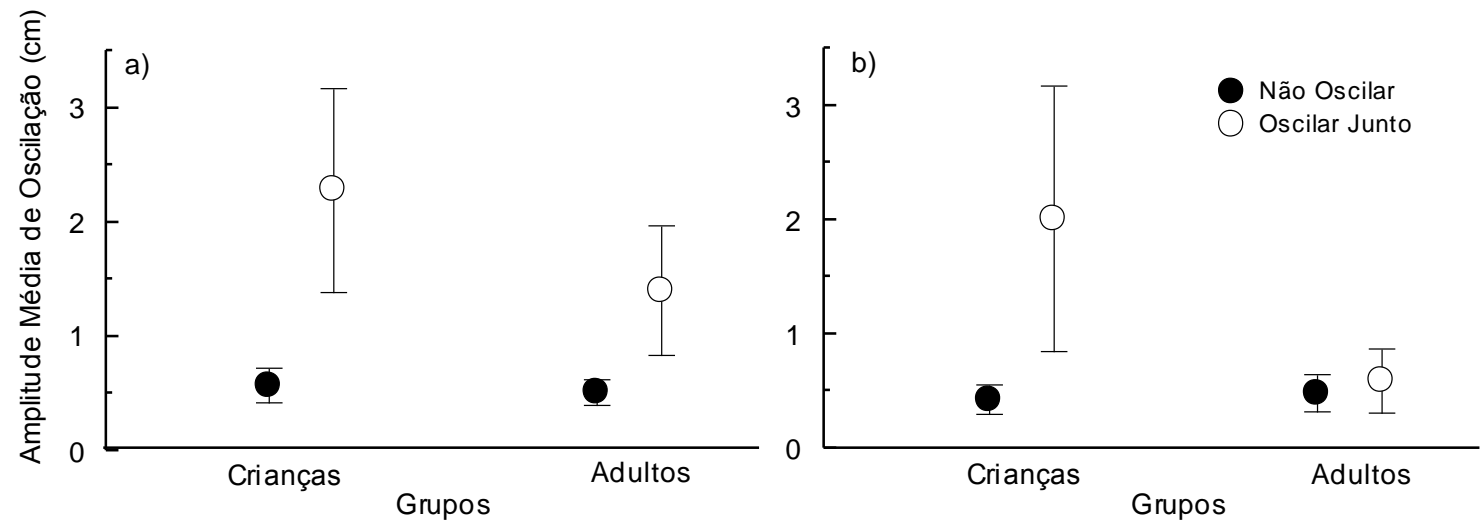

Figura 3. Média e desvio-padrão da amplitude média de oscilação para os grupos de crianças e adultos nas condições com informação correta (a) e com informação falsa (b) sobre o movimento da sala e com a solicitação de não oscilar (círculo cheio) e de oscilar junto (círculo aberto) com a sala móvel. 

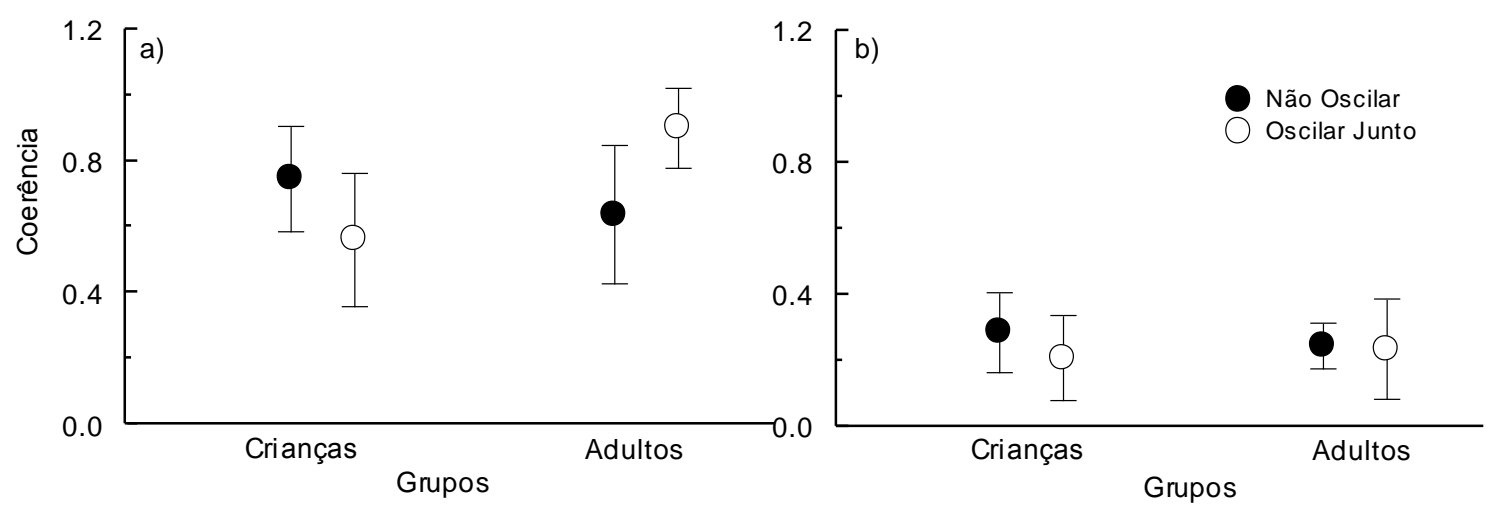

Figura 4. Média e desvio-padrão de coerência para os grupos de crianças e adultos nas condições com informação correta (a) e com informação falsa (b) sobre o movimento da sala e com a solicitação de não oscilar (círculo cheio) e de oscilar junto (círculo aberto) com a sala móvel.
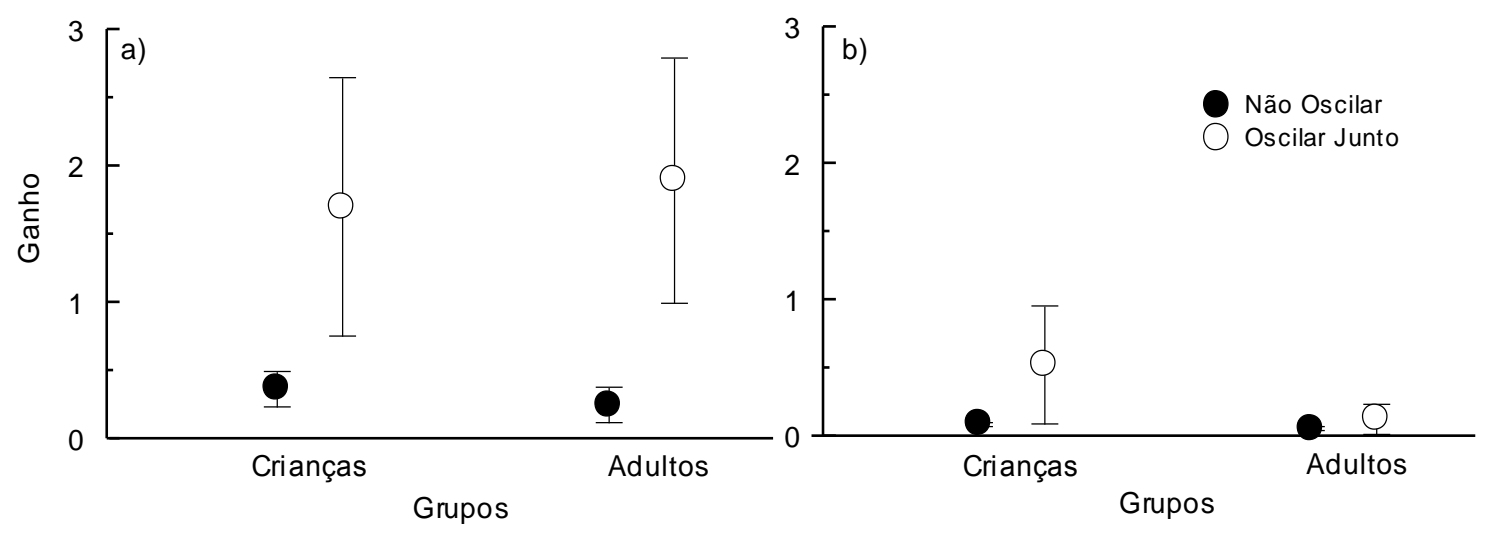

Figura 5. Média e desvio-padrão do ganho para os grupos de crianças e adultos nas condições com informação correta (a) e com informação falsa (b) sobre o movimento da sala e com a solicitação de não oscilar (círculo cheio) e de oscilar junto (círculo aberto) com a sala móvel.

O relacionamento espacial entre o movimento da sala $e$ as oscilações corporais com 0 fornecimento de informações corretas ou falsas na ação de não oscilar e oscilar junto com a sala móvel dos participantes foi verificado através das variáveis coerência e ganho. A Figura 4 apresenta os valores médios e respectivos desvios padrão da coerência nas duas condições, com 0 fornecimento de informação correta e falsa, para os dois grupos, na ação de não oscilar e oscilar junto com a sala. A Figura 5 apresenta os valores médios e respectivos desvios padrão do ganho nas duas condições, com o fornecimento de informação correta e falsa, para os dois grupos, na ação de não oscilar e oscilar junto com a sala.

A MANOVA revelou diferença para informação, Wilks` Lambda $=0,063$, $F(1,18)=125,83, \quad p<0,001$, ação, Wilks ' Lambda $=0,271, \quad F(1,18)=22,83, \quad p<0,001, \quad e$ interação entre grupo e ação, Wilks` Lambda $=0,515, \quad F(1,18)=8,01, \quad p<0,005, \quad e$ informação e ação, Wilks' Lambda $=0,327$, $F(1,18)=17,53, \quad p<0,001$. Análises univariadas indicaram que para o fator informação a diferença ocorreu para a coerência, $F(1,18)=219,72$, $p<0,001$, e para o ganho, $F(1,18)=79,42, p<0,001$. Em ambos os casos, oscilação corporal foi induzida e de forma mais coerente com o movimento da sala na condição de informação correta. Para o fator ação, a diferença foi observada apenas para o ganho, $F(1,18)=30,15$, $\mathrm{p}<0,001$, o mesmo ocorrendo para a interação informação e ação, $F(1,18)=25,90, \quad p<0,001$. Neste caso, a solicitação de oscilar junto levou maior oscilação corporal do que na ação de não oscilar e essa maior resposta ocorreu na condição de informação correta do que na condição de informação falsa. Finalmente, para a interação 
grupo e ação, a diferença ocorreu apenas para a coerência, $F(1,18)=9,27, p<0,01$, sendo que 0 grupo de adultos conseguiu produzir oscilação corporal mais coerente com o movimento da sala, quando solicitado para oscilar junto, e menos coerente com o movimento da sala, quando solicitado para não oscilar, quando comparado com o grupo de crianças.

\section{Discussão}

O objetivo do presente estudo foi examinar os efeitos da informação sobre o movimento da sala e da instrução em realizar uma ação motora na dinâmica intrínseca de funcionamento do sistema de controle postural em crianças e adultos. Os resultados mostraram que crianças apresentam maior oscilação corporal que adultos sem e com o movimento da sala móvel. Manipulação visual, propiciada pelo movimento do cenário visual, desencadeia movimento coerente em crianças e adultos, sendo que 0 relacionamento entre 0 movimento da sala móvel e a oscilação corporal foi similar entre ambos. Finalmente, a informação comportamental apresentada na forma de informação correta ou falsa sobre o movimento da sala e na forma de uma ação de oscilar ou não oscilar junto com a sala é utilizada de forma diferente por crianças para alterar a dinâmica intrínseca de funcionamento do sistema de controle postural, como discutido em parágrafos subsequentes. Este resultado sugere que crianças controlam suas ações motoras baseadas mais na dinâmica intrínseca de funcionamento do sistema do que nas informações provenientes de fontes externas.

A constatação de maior magnitude de oscilação corporal em crianças, sem e com a manipulação de informação visual a partir do movimento de uma sala móvel não é novidade, pois diversos estudos observaram tal comportamento (Schmuckler, 1997; Streepey e Angulo-Kinzler, 2002; Barela et al., 2003; Peterson et al., 2006; Godoi e Barela, 2008). Portanto, os resultados do presente estudo corroboram as observações prévias indicando que o funcionamento do sistema de controle postural de crianças, mesmo com idade de 8 anos, apresenta desempenho inferior do observado em adultos.

A constatação de que a manipulação da informação visual desencadeia oscilação corporal correspondente também não é novidade, pois diversos estudos observaram resultados similares em bebês (Lee e Aronson, 1974; Bertenthal e Bai, 1989; Barela, et al., 2000), crianças (Schmuckler, 1997; Godoi e Barela, 2008), adultos (Lee e Lishman, 1975; Barela, Barela et al., 2009) e idosos (Wade et al., 1995; Prioli et al., 2006). Entretanto, tem sido observado que 0 relacionamento entre informação visual e oscilação corporal é mais fraco e instável em crianças que em adultos, com crianças realizando oscilação que acompanhe precisamente a manipulação de estímulos sensoriais (Godoi e Barela, 2008; Barela et al., 2011) e, a primeira vista, os resultados deste estudo parecem contradizer os de estudos prévios. No presente estudo, os valores de coerência e ganho não diferiram entre crianças e adultos e a presente similaridade entre crianças e adultos pode decorrer de característica do estímulo proveniente do movimento da sala móvel. No presente estudo, o movimento da sala ocorreu com amplitude e velocidade $(1,8 \mathrm{~cm}$ e $0,9 \mathrm{~cm} / \mathrm{s})$, maiores que as anteriormente utilizadas (Godoi e Barela, 2008).

O uso de amplitude e velocidade maiores decorreu do fato de fornecer estímulo de forma a propiciar possível detecção consciente da manipulação visual, a partir do movimento da sala, para verificar 0 uso de informação comportamental por parte dos participantes. De qualquer forma, porém, pode-se sugerir, como ocorreu com o fornecimento de informação comportamental para adultos (Stoffregen et al., 2006; Barela, Barela et al., 2009), que o relacionamento entre informação visual e oscilação corporal é influenciado pelas características do estímulo fornecido. No presente estudo, o movimento da sala com amplitude e velocidade maiores possibilitou que as crianças não mais diferissem dos adultos, indicando que estímulos com características diferentes podem moldar a qualidade das respostas desencadeadas pelas manipulações.

Certamente, a observação mais importante foi a de que informação comportamental é utilizada diferentemente por crianças quando comparado com adultos. Vale ressaltar que informação sobre o movimento da sala ou solicitação de não oscilar não foram suficientes para suprimir os efeitos da manipulação da informação visual, provocado pelo movimento da sala, na oscilação corporal de crianças e adultos. Valores de amplitude média de oscilação (Fig. 3) e de coerência e ganho (Fig. 4) indicam que oscilação corporal, embora restrita, foi observada nas condições 
experimentais. Assim, mesmo com informação sobre o que estava ocorrendo ou sobre o que deveria fazer, quando ocorreu o movimento da sala, oscilação corporal correspondente foi desencadeada, indicando que a dinâmica intrínseca de adultos e crianças, envolvendo o relacionamento entre informação visual e oscilação corporal, não pode ser totalmente cancelada, pelo menos nas situações experimentais utilizadas no presente estudo.

Outra constatação importante foi que crianças e adultos apresentaram comportamentos diferentes quando informação correta e falsa, sobre o movimento da sala, foi apresentada e quando ocorreu solicitação de realizar a ação de não oscilar e oscilar junto com a sala. Os valores de amplitude média de oscilação indicam que, independente de a informação estar correta ou incorreta, a oscilação corporal desencadeada pela manipulação visual nas crianças foi similar nas situações experimentais utilizadas (Fig.3, painel a e b). Diferentemente, os adultos controlaram a magnitude de oscilação corporal de acordo com a informação apresentada. No caso de informação incorreta (Fig. 3, painel b), quando solicitados a oscilar junto com a sala, a magnitude de oscilação foi menor que a observada para a mesma solicitação com informação correta (Fig. 3, painel a). Constatação similar ocorre verificando os valores de coerência e ganho (Fig. 4 e Fig. 5, respectivamente). $\mathrm{Na}$ situação de informação correta, valores de coerência foram diferentes, com variação oposta, entre adultos e crianças para as solicitações de não oscilar e oscilar junto: crianças apresentaram valores de coerência maiores para a solicitação de não oscilar e menores para a solicitação de oscilar junto que adultos. Isso indica que crianças não seguiram tão bem a solicitação apresentada quando comparadas com adultos. $\mathrm{Na}$ situação de informação incorreta (Fig. 3, painel b), enquanto nenhuma diferença foi observada para adultos, quando solicitadas a não oscilar ou oscilar junto, crianças apresentaram valores de coerência maiores para a solicitação de não oscilar do que para oscilar junto.

Estes resultados indicam que crianças são capazes de alterar a influência de estímulos sensoriais provenientes do ambiente (dinâmica intrínseca - oscilação corporal desencadeada pelo movimento da sala), com base nas informações apresentadas ou pela solicitação de uma ação específica. Entretanto, crianças não são tão eficazes em alterar a dinâmica do relacionamento entre os estímulos sensoriais disponíveis e a ação motora realizada quando comparadas com adultos. Crianças são mais influenciadas pela informação proveniente do movimento da sala, independente da informação fornecida e da solicitação apresentada, do que adultos. Resultados similares foram recentemente observados quando crianças diminuíram a influência de estímulos sensoriais, quando os mesmos induzem oscilação corporal indesejável, porém a redução na influência sensorial foi menor que a observada para adultos (Rinaldi et al., 2009).

A adequação quanto ao uso de estímulos sensoriais mais informativos e úteis para a realização de uma ação motora específica decorre de um intrincado processo de repesagem sensorial (Carver, Kiemel et al., 2006; Barela et al., 2009) e que é calibrado ao longo da primeira década de vida (Rinaldi et al., 2009). Podemos acrescentar, ainda, que alterações decorrentes de informações sobre o contexto e de solicitação de uma ação específica parecem seguir o mesmo curso de desenvolvimento. No presente estudo, crianças foram mais influenciadas pelos estímulos sensoriais disponíveis do que adultos jovens, mesmo após serem solicitadas a alterar essa influência. Essa observação indica que crianças não conseguem se "desvencilhar" dos estímulos sensoriais disponíveis na mesma magnitude que adultos conseguem, sugerindo que a dinâmica intrínseca, que envolve o relacionamento entre informação sensorial e a realização de uma ação motora, pode ser alterada por informação e solicitação externa, porém ainda em menor magnitude que a observada em adultos.

Alteração das influências sensoriais é uma das características que propicia e garante flexibilidade na realização de ações motoras eficientes frente a um ambiente em constante alteração. Quando isso não ocorre, por exemplo, no caso da sala móvel, a orientação e o equilíbrio postural podem ser comprometidos, como observado nos estudos pioneiros de Lee e colaboradores (p.ex., Lee e Aronson, 1974) quando bebês tinham a postura ereta comprometida com a manipulação visual. Com base em estudos recentes em que a característica do estímulo sensorial foi manipulada (Godoi e Barela, 2008; Rinaldi et al., 2009), podemos sugerir que tal propriedade de funcionamento do sistema de controle postural é alterada ao longo da primeira década de vida, 
alcançando níveis observados em adultos apenas após os 12 anos de idade, quando ocorre a integração sensorial similar ao observado em adultos (Peterson et al., 2006). Parece que o mesmo ocorre com o uso de informação proveniente de fontes externas, tal como a informação sobre as condições ambientais e sobre uma ação a ser realizada.

A presente constatação traz implicações práticas importantes para a situação de ensinoaprendizagem envolvendo crianças. Os resultados do presente estudo indicam que crianças são mais dependentes da dinâmica intrínseca entre informação sensorial e oscilação corporal do que adultos jovens, de forma que elas são mais responsivas à manipulação visual e, consequentemente, apresentam resposta motora correspondente com maior magnitude. Considerando que a mudança de um dado comportamento, caracterizando uma situação de aprendizagem, implica em alterar a dinâmica intrínseca com base em objetivos comportamentais (Newell, 2003), seria importante e necessário criar situações que maximizem as informações apresentadas ou as ações solicitadas às crianças de forma que possíveis dificuldades na alteração da dinâmica intrínseca sejam minimizadas e a aprendizagem seja facilitada. Por exemplo, na situação de aprendizagem das três passadas do handebol, que pode ser confundida com as duas passadas na bandeja do basquetebol e que requer início com a perna esquerda/direita, respectivamente, para uma criança destra, seria interessante solicitar iniciar as passadas do handebol com uma passada mais forte, para marcar claramente com qual perna o movimento foi iniciado e, por conseguinte, ter clareza sobre a perna que iniciou a movimentação.

Diferentemente do observado anteriormente (Perotti Jr. et al., 2007), nenhuma diferença marcante foi observada entre a apresentação de informação sobre o contexto ambiental e a solicitação de uma ação específica. Assim, em uma situação de ensino-aprendizagem, o professor deve procurar fornecer informação sobre o contexto e solicitar uma ação para que possíveis alterações na dinâmica intrínseca sejam alcançadas. Tal discrepância entre os resultados anteriores e os do presente estudo pode decorrer do tipo de tarefa. Anteriormente a tarefa era discreta (Perotti Jr. et al., 2007) e, possivelmente, a solicitação de uma ação específica pode facilitar a execução da mesma em comparação com a apresentação de informação. No presente estudo, a tarefa implicou em um relacionamento contínuo e cíclico de informação sensorial ao longo de um período de tempo e, portanto, tanto informação sobre o contexto quanto sobre a ação a ser realizada deve ter contribuído para a realização da tarefa especifica. Entretanto, essa temática necessita ser melhor examinada para um entendimento mais aprofundado da mesma.

\section{Conclusão}

A dinâmica intrínseca envolvendo o relacionamento entre informação sensorial e oscilação corporal pode ser alterada por informação sobre o contexto ambiental e sobre uma ação a ser realizada tanto em crianças quanto em adultos, em tarefas que envolvam o uso cíclico de informação sensorial. Apesar de serem capazes de alterar a dinâmica intrínseca, crianças não conseguem assim o fazer com a mesma magnitude que adultos, observada por maior indução de oscilação corporal com a manipulação visual, indicando que elas são mais dependentes da dinâmica intrínseca do que adultos. Essa constatação traz implicações importantes para a situação de ensinoaprendizagem, pois indica que situações de aprendizagem envolvendo crianças devem ser estruturadas propiciando condições mais favoráveis para alterações na dinâmica intrínseca de funcionamento para que os objetivos da mesma sejam alcançados.

\section{Referências}

Barela, A. M.; Barela, J. A.; Rinaldi, N. M.; Toledo, D. R. Influence of imposed optic flow characteristics and intention on postural responses. Motor Control, Champaign, v.13, p.119-129, 2009.

Barela, J. A.; Barela, A. M. F. O contexto da aprendizagem motora: perspectivas teóricas e desafios metodológicos na abordagem dos sistemas dinâmicos. In: M. D. G. S. Guedes (Ed.). Aprendizagem Motora: problemas e contextos. Cruz Quebrada: Faculdade de Motricidade Humana, 2001, p.59-69.

Barela, J. A.; Freitas Júnior, P. B.; Godoi, D.; Polastri, P. F. The acquisition of siting position in infants: The coupling between visual information and trunk sway. In: J. V. D. Kamp, A. Ledebt, et al (Ed.). Advances in motor development and learning in infacy. Enschede: Printpartners Ipskamp, 2001, p.23-26. 
Barela, J. A.; Godoi, D.; Freitas Junior, P.; Polastri, P. F. Visual information and body sway coupling in infants during sitting acquisition. Infant Behavior \& Development, Norwood, v.23, p.285297, 2000.

Barela, J. A.; Jeka, J. J.; Clark, J. E. Postural control in children: Coupling to dynamic somatosensory information. Experimental Brain Research, Amsterdam, v.150, p.434-442, 2003.

Barela, J. A.; Sanches, M.; Lopes, A. G.; Razuk, M.; Moraes, R. Use of monocular and binocular visual cues for postural control in children.

Journal of Vision, Charlottesville, v.11, n.12, p.10, 1-8, 2011.

Barela, J. A.; Toledo, D. R. D.; Ferreira, D. M. A.; Polastri, P. F. Repesagem e adaptação sensorial no controle postural de adultos. Neurociências, Rio de Janeiro, v.5, n.3, p.141-149, 2009.

Bertenthal, B. I.; Bai, D. L. Infant's sensitivity to optical flow for controlling posture.

Developmental Psychology, Washington, v.25, n.6, p.936-945, 1989.

Bertenthal, B. I.; Rose, J. L.; Bai, D. L. Perceptionaction coupling in the development of visual control of posture. Journal of Experimental Psychology: Human Perception and Performance, Washington, v.23, n.6, p.16311643, 1997.

Butterworth, G.; Hicks, L. Visual proprioception and postural stability in infancy. A developmental study. Perception, London, v.6, p. 256-262, 1977.

Carver, S.; Kiemel, T.; Jeka, J. J. Modeling the dynamics of sensory reweighting. Biological Cybernetics, Berlin, v.95, n.2, p.123-134, 2006.

Delorme, A.; Frigon, J.; Grothe, M. Postural reactions to a moving environment: Influence of aging in adults. In: B. G. Bardy, R. J. Bootsma, et al (Ed.). Studies in perception and action III. Hillsdale, NJ: LEA, 1995, p.7-10.

Freitas Júnior, P. B.; Barela, J. A. Postural control as a function of self-and object-motion perception. Neuroscience Letters, Amsterdam, v.369, p.6468, 2004.

Godoi, D.; Barela, J. A. Body sway and sensory motor coupling adaptation in children: Effects of distance manipulation. Developmental

Psychobiology, New York, v.50, p.77-87, 2008.

Higgins, C. I.; Campos, J. J.; Kermoian, R. Effect of self-produced locomotion on infant postural compensation to optic flow. Developmental Psychology, Washington, v.32, n.5, p.836-841, 1996.
Jeka, J. J.; Schöner, G.; Dijkstra, T.; Ribeiro, P.; Lackner, J. R. Coupling of fingertip somatosensory information to head and body sway. Experimental Brain Research, Amsterdam, v.113, p.475-483, 1997.

Lee, D. N.; Aronson, E. Visual proprioceptive control of standing in human infants. Perception and Psychophysics, Austin, v.15, n.3, p. 529532, 1974

Lee, D. N.; Lishman, J. R. Visual proprioceptive control of stance. Journal of Human Movement Studies, Amsterdam, v.1, p.87-95, 1975.

Lishman, J. R.; Lee, D. N. The autonomy of visual kinaesthesis. Perception, London, v.2, p.287-294, 1973.

Newell, K. M. Change in motor learning: A coordination and control perspective. Motriz, Rio Claro, v.9, n.1, p.1-6, 2003.

Perotti Jr., A.; Barela, J. A.; Polastri, P. F.; Tani, G. Influência de diferentes informações comportamentais na dinâmica intrínseca entre informação visual e oscilação corporal. Brazilian Journal of Motor Behavior, Rio Claro, v.2, n.1, p.40-50, 2007.

Peterson, M. L.; Christou, E.; Rosengren, K. S. Children achieve adult-like sensory integration during stance at 12-years-old. Gait \& Posture, Oxford, v.23, p.455-463, 2006.

Prioli, A. C.; Cardozo, A. S.; Júnior, P. B. D. F.; Barela, J. A. Task demand effects on postural control in older adults. Human Movement Science, Amsterdam, v.25, p.435-446, 2006.

Rinaldi, N. M.; Polastri, P. F.; Barela, J. A. Agerelated changes in postural control sensory reweighting. Neuroscience Letters, Amsterdam, v.467, p.225-229, 2009.

Schmuckler, M. A. Children's postural sway in response to low- and high-frequency visual information for oscillation. Journal of Experimental Psychology: Human Perception and Performance, Washington, ,v.23, n.2, p.528545, 1997.

Schöner, G.; Dijkstra, T. M. H.; Jeka, J. J. Actionperception patterns emerge from coupling and adaptation. Ecological Psychology, Hillsdale, v.10, n.3-4, p.323-346, 1998.

Schöner, G.; Kelso, J. A. S. A synergetic theory of environmentally-specified and learned patterns of movement coordination. I. Relative phase dynamics. Biological Cybernetics, Berlin, v.58, p.71-80, 1988a. 
Schöner, G.; Kelso, J. A. S . A synergetic theory of environmentally-specified and learned patterns of movement coordination. II. Component oscillator dynamics. Biological Cybernetics, Berlin, v.58, p.81-89, 1988b.

Schöner, G.; Zanone, P. G.; Kelso, J. A. S.

Learning as change of coordination: Theory and experiment. Journal of Motor Behavior, Washington, v.24, n.1, p.29-48, 1992.

Soames, R. W.; Atha, J. The spectral characteristics o postural sway behavior. European Journal of Applied Physiology, Berlin, v.49, p.169-177, 1982.

Stoffregen, T. A.; Hove, P.; Schmit, J.; Bardy, B. G. Voluntary and involuntary postural responses to imposed optic flow. Motor Control, Champaign, v.10, p.24-33, 2006.

Streepey, J. W.; Angulo-Kinzler, R. M. The role of task difficulty in the control of dynamic balance in children and adultss. Human Movement Science, Amsterdam, v.21, p.423-438, 2002.

Wade, M. G.; Lindquist, R.; Taylor, J. R.; TreatJacobson, D. Optical flow, spatial orientation, and the control of posture in the elderly. Journal of Gerontology, Washington, v.50B, n.1, p.P51P58, 1995.

Zanone, P. G.; Kelso, J. A. S. Learning and transfer as dynamical paradigms for behavioral change. In: G. E. Stelmach e J. Requin (Ed.).

Tutorials in Motor Behavior II. New York:

Elsevier Science Publishers B.V., 1992, p.563582.

\section{Endereço:}

Alaércio Perotti Junior Centro Universitário Hermínio Ometto Av. Dr. Maximiliano Baruto, 500 Jardim Universitário, Araras, SP, Brasil 13607-339

Telefone: (19) 3543-1400

e-mail: alaercioperotti@gmail.com

Recebido em: 17 de março de 2012. Aceito em: 8 de setembro de 2012.

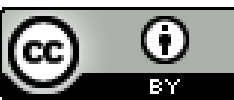

Motriz. Revista de Educação Física. UNESP, Rio Claro, SP, Brasil - elSSN: 1980-6574 - está licenciada sob Creative Commons - Atribuição 3.0 